\title{
Conserved Functional Surface of Antimammalian Scorpion $\beta$-Toxins
}

\author{
Rong Chen* and Shin-Ho Chung \\ Research School of Biology, Australian National University, Canberra, ACT 0200, Australia
}

ABSTRACT: Scorpion $\beta$-toxins bind to the voltage-sensing domain of voltage-gated sodium $\left(\mathrm{Na}_{\mathrm{V}}\right)$ channels and trap the voltage-sensing domain in the activated state. Two structurally similar $\beta$-toxins from scorpions, Css 4 and $\mathrm{Cn} 2$, selectively target different subtypes of mammalian $\mathrm{Na}_{\mathrm{V}}$ channels. While the receptor site on the channels is known, the functional surface of the toxins remains to be understood. Here, we predict the binding modes of Css 4 and $\mathrm{Cn} 2$ to the voltage-sensing domains of $\mathrm{Na}_{\mathrm{V}} 1.2$ and $\mathrm{Na}_{\mathrm{V}} 1.6$, respectively, with a molecular docking method and molecular dynamics simulations. The dissociation constants for the predicted toxin-channel complexes derived with umbrella sampling simulations are in accord with experiment. Our calculations suggest that the functional surface of $\mathrm{Cn} 2$ and Css 4 is primarily formed by the loop between positions 8 and 18, centered on the two charged residues Lys13 and Glu15.

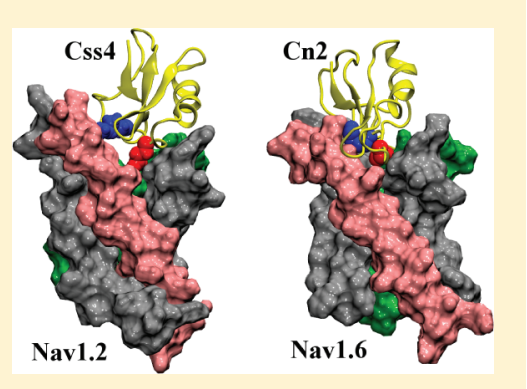

\section{INTRODUCTION}

Voltage-gated sodium $\left(\mathrm{Na}_{\mathrm{V}}\right)$ channels contribute to the rising phase of action potential. Various $\mathrm{Na}_{\mathrm{V}}$ channel isoforms of similar functional properties but different distribution in mammalian muscle and nerve cells have been identified. ${ }^{1} \mathrm{Na}_{\mathrm{V}}$ channels have also been discovered in bacteria. ${ }^{2,3}$ Bacterial $\mathrm{Na}_{\mathrm{V}}$ channels such as the one from Arcobacter butzleri $\left(\mathrm{Na}_{\mathrm{V}} \mathrm{Ab}\right)$, whose crystal structure has been solved, ${ }^{4}$ are homotetramers, with each monomer consisting of six transmembrane helices S1-S6. The four helices S1-S4 of each subunit form a voltagesensing (VS) domain, whereas the S5-S6 helices of the four subunits line the pore domain. Mammalian $\mathrm{Na}_{\mathrm{V}}$ channels, on the other hand, are hetro-tetramers consisting of four nonidentical subunits. ${ }^{5}$

Scorpion $\beta$-toxins are short polypeptides containing $60-80$ amino acids, whose backbones are cross-linked by four disulfide bridges. ${ }^{5-7}$ Different $\beta$-toxins specifically targeting mammalian or insect $\mathrm{Na}_{V}$ channels have been identified. ${ }^{6}$ Antimammalian $\beta$-toxins such as $\mathrm{Cn} 2$ and Css 4 selectively target $\mathrm{Na}_{\mathrm{V}}$ channels of mammals. $\mathrm{Cn} 2$ isolated from Centruroides noxius ${ }^{8}$ is specific for $\mathrm{Na}_{\mathrm{V}} 1.6$ channels, ${ }^{9}$ whereas Css 4 isolated from Centruroides suffusus suffusus ${ }^{10}$ is sensitive to both $\mathrm{Na}_{\mathrm{V}} 1.6$ and $\mathrm{Na}_{\mathrm{V}} 1.2$ channels. ${ }^{9,11}$ The two toxins share $83 \%$ sequence identity (Figure 1A). Antimammalian $\beta$-toxins bind to the periplasmic top of the VS domain of the II subunit of $\mathrm{Na}_{\mathrm{V}}$ channels and interfere with channel activation. ${ }^{9,11-17}$ Following a transient depolarization voltage, the addition of $\beta$-toxins can cause sensitive $\mathrm{Na}_{V}$ channels to open at less depolarized membrane potentials, leading to a left-shifted activity-voltage curve. ${ }^{5}$ The VS-trapping model has been proposed to describe the mechanism of action by $\beta$-toxins. ${ }^{13}$ According to this model, the toxins bind to the II VS domain (IIS1-S4) in the channel and stabilize the VS domain in the activated state. However, growing experimental evidence suggests that the pore loop of the III subunit, spatially in close proximity to the VS domain of the II subunit, ${ }^{4,18}$ may also be involved in the binding of scorpion $\beta$-toxins. ${ }^{19-21}$
The functional surface of antimammalian $\beta$-toxins has not been resolved, although several mutagenesis experiments have been carried out. ${ }^{15,17,22}$ These experiments have suggested that two glutamate residues at positions 15 and 28 and several neutral residues at positions 19, 22, 24, 40, 42, and 44 of Css 4 are important for the binding and function of the toxin. It has been suggested that the receptor site of Css 4 on the $\mathrm{Na}_{\mathrm{V}} 1.2$ channel contains 10 important residues from the $\mathrm{S} 1-\mathrm{S} 2$ and S3-S4 linkers, including three glutamates at positions 779,837 , and $844 .{ }^{11}$ On the basis of these findings, however, the toxinchannel interacting residue pairs cannot be readily identified, because the known possible key residues of both the toxin functional surface and the channel receptor site comprise only neutral and acidic residues. From an electrostatic point of view, we would expect that some of the basic and acidic residues of the toxin and the channel are also involved in binding (Figure 2).

Here, with a molecular docking method and molecular dynamics (MD) simulations, we investigate the binding of the $\beta$-toxins Css 4 and $\mathrm{Cn} 2$ to the II VS domains of $\mathrm{Na}_{\mathrm{V}} 1.2$ and $\mathrm{Na}_{\mathrm{V}} 1.6$, respectively. We show that the loops between positions 8 and 18 of the two toxins wedge into the receptor site formed by the S1-S2 and S3-S4 linkers of the VS domains. The binding modes we uncovered are consistent with a wide range of experimental data and suggest the key roles of two charged residues, Lys13 and Glu15, which are conserved between antimammalian $\beta$-toxins, in the binding to $\mathrm{Na}_{\mathrm{V}}$ channels.

\section{COMPUTATIONAL METHODS}

Homology Models. We construct atomic models of the isolated IIS1-S4 domains of rat $\mathrm{Na}_{\mathrm{V}} 1.2$ (NCBI entry $\mathrm{NP}$ _036779.1) and rat $\mathrm{Na}_{\mathrm{V}} 1.6$ (NCBI entry AAC42059.1) with the homology modeling server SWISS-MODEL, ${ }^{23-25}$

Received: January 4, 2012

Revised: March 23, 2012

Published: April 3, 2012 
A

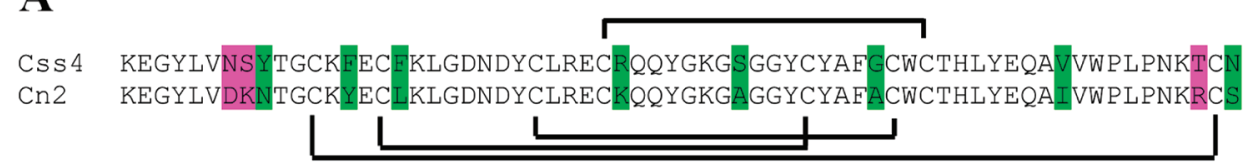

B

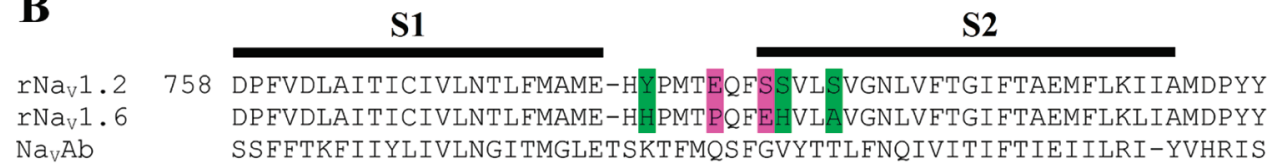

$\mathrm{rNa}_{V} 1.2818$ YFQEGWNIFDGFIVSLSLMELGLANVEGLSVLRSFRLLRVFKLAKSWPTLNMLIKIIGNS

$r N a_{V} 1.6$ YFQEGWNIFDGFIVSLSLMELSLADVEGLSVLRSFRLLRVFKLAKSWPTLNMLIKI IGNS

$\mathrm{Na}_{\mathrm{V}} \mathrm{Ab} \quad$ FFKDPWSLFDFFVVAISL----VPTSSGFEILRVLRVLRLFRLVTAVPQMRKIVSALISV

S3

S4

Figure 1. (A) Sequence alignment of the $\beta$-toxins Css4 and Cn2, showing an identity of $83 \%$. Key different residues are highlighted in purple, and other different residues are in green. Horizontal lines indicate disulfide bridges. (B) Sequence alignment of the IIS1-S4 domains of rat Na 1.2 and $\mathrm{Na}_{\mathrm{V}} 1.6$ channels. The sequence of $\mathrm{Na}_{\mathrm{V}} \mathrm{Ab}$ is also shown. Horizontal bars indicate the four transmembrane helices S1-S4. Numbering is that of $\mathrm{Na}_{\mathrm{V}} 1.2$.

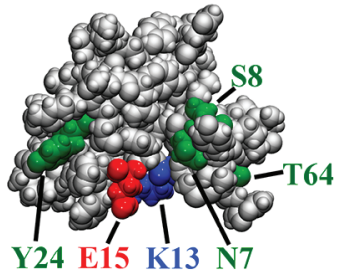

Css4

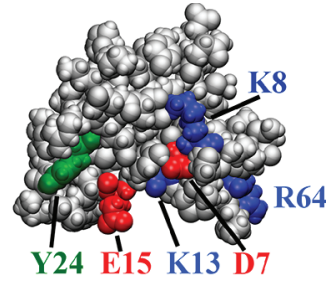

$\operatorname{Cn} 2$

Figure 2. Molecular surfaces of the $\beta$-toxins Css4 (left) and $\mathrm{Cn} 2$ (right). The important residues in forming the toxin-VS complexes are shown in red (acidic residues), blue (basic residues), and green (neutral residues).

using the crystal structure of the bacterial $\mathrm{Na}_{\mathrm{V}}$ channel $\mathrm{Na}_{\mathrm{V}} \mathrm{Ab}$ as a template. ${ }^{4}$ The II VS domains of $\mathrm{Na}_{\mathrm{V}} 1.2$ and $\mathrm{Na}_{\mathrm{V}} 1.6$ differ in only seven residues, primarily located at the S1-S2 and S3S4 linkers (Figure 1B). Sequence alignment of $\mathrm{Na}_{\mathrm{V}} 1.2$ and $\mathrm{Na}_{V} 1.6$ with $\mathrm{Na}_{V} \mathrm{Ab}$ shows a gap of four residues in the S3-S4 linker region of the II VS domain (Figure 1B). Thus, homology models of the II VS domain of both $\mathrm{Na}_{\mathrm{V}} 1.2$ and $\mathrm{Na}_{\mathrm{V}} 1.6$ channels could be readily generated. However, high-quality homology models of the pore domain could not be obtained, due to the lack of high sequence similarity between bacterial and mammalian $\mathrm{Na}_{\mathrm{V}}$ channels. Therefore, the interactions between the toxins and the III pore domain of the channels are not taken into account in the present work. We also construct a structural model of Css4 using the solution structure of $\mathrm{Cn} 2$ (PDB ID $1 \mathrm{CN} 2)^{26}$ as a template.

Molecular Docking. We use molecular docking calculations to generate the most plausible bound complex between each toxin-VS pair (Css4- $\mathrm{Na}_{\mathrm{V}} 1.2$ and $\mathrm{Cn} 2-\mathrm{Na}_{\mathrm{V}} 1.6$ ), which is used as the starting configuration of subsequent unbiased $\mathrm{MD}$ simulations. Ideally, the toxin should be released in water and allowed to bind to the VS domain spontaneously in the MD simulations. However, such spontaneous binding requires a time scale beyond what can be achieved with the MD simulation technique. Thus, we use docking calculations to generate crude bound complexes, which can then be refined with MD simulations.

We dock Css 4 to the isolated IIS1-S4 domain of $\mathrm{Na}_{\mathrm{V}} 1.2$ and $\mathrm{Cn} 2$ to the IIS1-S4 domain of $\mathrm{Na}_{\mathrm{V}} 1.6$ with the rigid-body docking program ZDOCK 3.0.1. ${ }^{27}$ For each toxin-VS pair, 300 configurations are generated. We note that the flexibility of both the toxin and the VS domain is ignored in the docking calculations. In addition, the docking calculations are performed without the presence of the lipids, water, and ions. Thus, we have expected and indeed found that most of the configurations generated are unrealistic binding modes. We select the most plausible configurations according to experimental data rather than docking scores. A configuration is considered to be plausible if the residues at positions 7,8 , and 64 of the toxin are in close proximity to the VS domain. These three positions are likely to be directly involved in binding, because differences in these positions render the distinct sensitivities of Css 4 and $\mathrm{Cn} 2$ to $\mathrm{Na}_{\mathrm{V}}$ channels observed experimentally. ${ }^{9}$ In each case, we only find one unique plausible configuration, which is ranked between 200 and 300, indicating that the docking method is grossly adequate in predicting the bound states of the toxins.

Molecular Dynamics Simulations. Each selected toxinVS complex (Css4- $\mathrm{Na}_{\mathrm{V}} 1.2$ and $\left.\mathrm{Cn} 2-\mathrm{Na}_{\mathrm{V}} 1.6\right)$ is embedded in a POPC (2-oleoyl-1-palmitoyl-sn-glycero-3-phosphocholine) bilayer and a box of explicit water containing $0.2 \mathrm{M} \mathrm{NaCl}$. The systems, consisting of the toxin-VS complex, the POPC bilayer $(\sim 80$ lipids/leaflet), $\sim 16700$ water molecules, and $63 \mathrm{NaCl}$, are $\sim 77 \times 77 \times 123 \AA^{3}$ in size. MD simulations are then carried out to allow the systems to evolve to a thermodynamically stable state. Each system is simulated twice with different random initial velocities, each lasting $20 \mathrm{~ns}$. Similar toxin-VS interacting residue pairs are observed from the two simulations.

All molecular dynamics simulations are performed using NAMD $2.8^{28}$ at 1 atm and $300 \mathrm{~K}$, with periodic boundary conditions and a 2 -fs time step. The CHARMM36 force field is used to describe the interatomic interactions of lipids, proteins, and ions. ${ }^{29,30}$ The TIP3P model ${ }^{31}$ is used to describe water molecules. The switch and cutoff distances for short-range interactions are set to 8.0 and $12.0 \AA$, respectively. The particle mesh Ewald method is used to describe long-range electrostatic interactions, with a maximum grid spacing of $1.0 \AA$. The SHAKE $^{32}$ and SETTLE ${ }^{33}$ algorithms are used to maintain rigid bond lengths. Trajectories are saved every 20 ps for analysis. 
Umbrella Sampling. To measure the dissociation constant for the binding of the toxins to the VS domains, we construct one-dimensional potential of mean force (PMF) profiles for the unbinding of the toxins along the channel axis parallel to the bilayer normal ( $z$ dimension). For each bound complex, a force of $20 \mathrm{kcal} / \mathrm{mol} / \AA$ is applied to pull the toxin out from the binding site along the channel axis. The toxin backbone is maintained rigid using harmonic restraints during the pulling, whereas the backbone atoms of the channel are fixed. The pulling generates the starting structures for the umbrella windows spaced at $0.5 \AA$ intervals. In subsequent umbrella sampling simulations, the $\mathrm{C} \alpha$ atoms of the VS domains are harmonically restrained with a force constant of $0.25 \mathrm{kcal} / \mathrm{mol} /$ $\AA^{2}$, allowing faster convergence of the PMF. Here, we assume that no major changes in the conformation of the VS domains are induced by toxin binding, because the maximum rmsd of the VS backbone with reference to the starting structure is $<2.5$ $\AA$ for $\mathrm{Na}_{\mathrm{V}} 1.2$ and $<3.0 \AA$ for $\mathrm{Na}_{\mathrm{V}} 1.6$, during the 20 -ns simulations of the bound complexes.

The center of mass (COM) of the toxin backbone is restrained to the center of each umbrella window using a harmonic force constant of $30 \mathrm{kcal} / \mathrm{mol} / \AA^{2}$, unless otherwise stated. To improve convergence and reduce sampling errors, ${ }^{34}$ the force constant for $\mathrm{Cn} 2$ at the window $z=29.5 \AA$ is increased to $50 \mathrm{kcal} / \mathrm{mol} / \AA^{2}$, and two extra windows are added at $z=29.3 \AA$ and $z=29.7 \AA$. For Css 4 , the force constant for the windows between $z=26.5 \AA$ and $z=28.0 \AA$ is increased to $40 \mathrm{kcal} / \mathrm{mol} / \AA^{2}$. The COM of the VS domain is at $z=0 \AA$. The $\mathrm{COM}$ of the toxin backbone is restrained in a cylinder of $8 \AA$ in radius centered on the channel axis, using a flat-bottom harmonic restraint $\left(20 \mathrm{kcal} / \mathrm{mol} / \AA^{2}\right)$. This radius of $8 \AA$ is chosen such that the restraint is not felt by the toxin when it is bound. Each umbrella window is simulated for at least 5 ns until convergence is obtained. We assume a convergence if the depth of the PMF profile changes by $<0.5 \mathrm{kT}$ over the last $1 \mathrm{~ns}$. The first $1 \mathrm{~ns}$ of each window, considered as equilibration, is removed from data analysis. The COM coordinate of the toxin along the $z$ dimension is saved every 1 ps for analysis. The weighted histogram analysis method is used to construct the PMF profile, ${ }^{35}$ based on which a dissociation constant $\left(K_{d}\right)$ is derived using the following equation: ${ }^{36}$

$$
K_{\mathrm{d}}{ }^{-1}=1000 \pi R^{2} N_{\mathrm{A}} \int_{z_{\min }}^{z_{\max }} \exp [-W(z) / k T] d z
$$

where $R$ is the radius of the cylinder ( $8 \AA$ ), $N_{\mathrm{A}}$ is Avogadro's number, $z_{\min }=24 \AA$ and $z_{\max }=45 \AA$ are the boundaries of the binding site along the reaction coordinate $(z), W(z)$ is the PMF, $k$ is the Boltzmann constant, and $T$ is the temperature. The window $z=45 \AA$ is considered as bulk, the PMF of which is set to zero. The statistical error of the PMF profile is estimated with a bootstrapping method. From trajectories of the umbrella sampling simulations, 20 sets of data points $(z$ coordinates) are randomly generated, allowing repetitive sampling. For each of the 20 trajectories, a PMF profile is constructed, resulting in $20 \mathrm{PMF}$ profiles. The standard deviation of the PMF as a function of $z$ is calculated, which is zero at the reference point where the PMF is set to a constant of zero and accumulates to a maximum along the PMF profile.

\section{RESULTS AND DISCUSSION}

Binding of Css4 to $\mathrm{Na}_{\mathrm{v}}$ 1.2. It has been shown experimentally that Css4 binds to the S1-S2 and S3-S4 linkers of the VS domain of $\mathrm{Na}_{\mathrm{V}} 1.2$ and shifts the activityvoltage curve to the left. ${ }^{11}$ With docking calculations and MD simulations, we observe that the loop between positions 8 and 18 of the toxin wedges into the binding groove formed by the S1-S2 and S3-S4 linkers. The binding is stabilized by favorable nonbonded interactions between the toxin and the VS domain.

The position of Css 4 bound to the IIS1-S4 domain of $\mathrm{Na}_{\mathrm{V}} 1.2$ relative to the lipid bilayer after $20 \mathrm{~ns}$ of unbiased $\mathrm{MD}$ simulation is shown in Figure 3A. It can be seen that Css 4 is
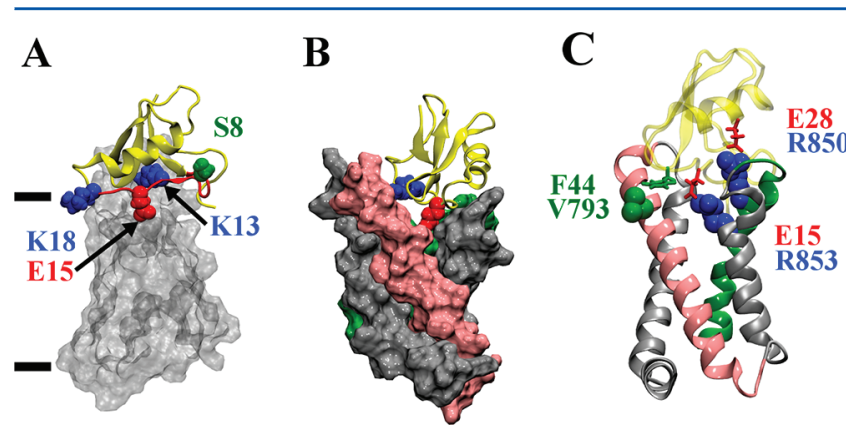

Figure 3. (A) Position of Css4 bound to the IIS1-S4 VS domain of $\mathrm{Na}_{\mathrm{V}} 1.2$, relative to the lipid bilayer. The surface of the VS domain is shown in transparent silver. Toxin backbone is in yellow. Horizontal lines indicate the average position of the phosphorus atoms of the lipid head groups. The loop between positions 8 and 18 of the toxin is highlighted in red. (B) Css4 bound to the VS domain of $\mathrm{Na}_{\mathrm{V}} 1.2$. The side chains of two key residues of the toxin, Lys13 (blue) and Glu15 (red), are highlighted. The S2 and S4 helices of $\mathrm{Na}_{\mathrm{V}} 1.2$ are highlighted in pink and lime, respectively. (C) Css4 bound to the VS domain of $\mathrm{Na}_{V} 1.2$ showing three of the key contacts. Toxin backbone is shown in yellow. The complexes shown in panels $\mathrm{B}$ and $\mathrm{C}$ in this and the following figure are rotated by approximately $90^{\circ}$ counterclockwise from that of panel $\mathrm{A}$.

indeed attracted to the periplasmic side of the VS domain, consistent with the receptor site for $\beta$-toxins previously suggested. ${ }^{11}$ The two charged residues, Lys 13 and Glu15, penetrate deeply into the binding groove between the S1-S2 and S3-S4 linkers (Figure 3B). These two residues are at the center of the loop between positions 8 and 18 of the toxin, as indicated in Figure 3A. Table 1 tabulates the key residue pairs identified from the bound complex and the average distances between these residue pairs over the last $15 \mathrm{~ns}$ of the sampling period. The toxin residues Lys13, Glu15, and Glu28 are

Table 1. Interaction Residue Pairs between Css4 and $\mathrm{Na}_{\mathrm{V}} 1.2$ and between $\mathrm{Cn} 2$ and $\mathrm{Na}_{\mathrm{V}} 1.6$ in the Bound States of the Toxins, Obtained after $20 \mathrm{~ns}$ of Simulation ${ }^{a}$

\begin{tabular}{|c|c|c|c|}
\hline Css4-Na 1.2 & av distance & $\mathrm{Cn} 2-\mathrm{Na}_{\mathrm{V}} 1.6$ & av distance \\
\hline N7-S851 & $2.2 \pm 0.3$ & D7-R850 & $1.9 \pm 0.4$ \\
\hline K13-E779 & $2.5 \pm 1.0$ & K13-E779 & $3.8 \pm 0.4$ \\
\hline K13-S788 & $3.5 \pm 1.0$ & K13-E788 & $2.3 \pm 0.3$ \\
\hline E15-R853 & $3.4 \pm 0.9$ & E15-R853 & $1.7 \pm 0.1$ \\
\hline Y24-V843 & $2.9 \pm 0.5$ & Y24-V843 & $2.4 \pm 0.4$ \\
\hline E28-R850 & $1.7 \pm 0.2$ & E28-E844 & $5.1 \pm 1.3$ \\
\hline F44-V793 & $2.8 \pm 0.4$ & F44-L796 & $2.2 \pm 0.3$ \\
\hline T64-H780 & $2.4 \pm 0.4$ & R64-E779 & $3.1 \pm 0.4$ \\
\hline
\end{tabular}

${ }^{a}$ Numbering of channel is that of $\mathrm{Na}_{\mathrm{V}} 1.2$. The minimum distances $(\AA)$ of each residue pair including hydrogen atoms averaged over the last $15 \mathrm{~ns}$ are given. Standard deviations are also shown. 
observed to form electrostatic complexes with several charged residues of the VS, whereas the residues Tyr24 and Phe44 form hydrophobic clusters with several hydrophobic residues of the VS (Figure $3 \mathrm{C}$ ). The Css $4-\mathrm{Na}_{\mathrm{V}} 1.2$ complex is very stable in the sampling period of $20 \mathrm{~ns}$, as reflected in the standard deviations of the distances, which are $\leq 1.0 \AA$ for all of the interacting residue pairs (Table 1 ). Thus, Css 4 binds tightly to $\mathrm{Na}_{\mathrm{V}} 1.2$ by electrostatic and hydrophobic interactions with the channel. Our calculations reveal that the functional surface of Css 4 is primarily formed by the loop between positions 8 and 18 of the toxin, centered on the two charged residues Lys 13 and Glu15, which are conserved in antimammalian scorpion $\beta$-toxins. ${ }^{6}$

The binding mode between Css 4 and $\mathrm{Na}_{\mathrm{V}} 1.2$ we predicted is consistent with experiment. For example, the mutation to glutamine of the residue Glu779, in contact with the toxin residue Lys13 in our model, completely abolishes the ability of Css4 in trapping the VS of $\mathrm{Na}_{\mathrm{V}} 1.2{ }^{16}$ The two arginine residues at positions 850 and 853 of the S4 helices, experimentally shown to be important for the activity of Css $4,{ }^{16,37}$ are observed to form electrostatic complexes with two glutamate residues of Css4 (Table 1). In addition, the functional surface of Css4 we observed is consistent with the mutagenesis experiments of Cohen et al. ${ }^{15}$ for Css 4 and Karbat et al. ${ }^{38}$ for the anti-insect $\beta$-toxin LqhIT2. For example, the residues Tyr24, Glu28, and Phe44 of Css4, the single mutation of which to alanine causes more than 100 -fold reduction in binding affinity according to the mutagenesis experiments of Cohen et al., ${ }^{15}$ are observed to be in direct contact with the channel (Table 1). However, we note that mutagenesis data should be interpreted with caution, as both the conformation and the binding mode of the toxin could be altered by mutation.

Our model is also in agreement with the models of Css4$\mathrm{Na}_{\mathrm{V}} 1.2$ proposed by Catterall and co-workers, ${ }^{11,16}$ in which the two key acidic residues, Glu15 and Glu28, are in close proximity to the receptor site. However, the role of Lys 13 in binding and the toxin-VS interacting residue pairs are not clear in the previous models. ${ }^{11,16}$

Binding of $\mathrm{Cn} 2$ to $\mathrm{Na}_{\mathrm{v}}$ 1.6. $\mathrm{Cn} 2$ has been observed to shift the activity-voltage curve of $\mathrm{Na}_{\mathrm{V}} 1.6$ to the left. ${ }^{9}$ Here, we show that, similar to Css4, $\mathrm{Cn} 2$ also wedges into the binding groove on $\mathrm{Na}_{\mathrm{V}} 1.6$ with the 8-18 loop centered on Lys13 and Glu15 and forms a stable complex with the channel.

The position of $\mathrm{Cn} 2$ bound to the IIS1-S4 domain of $\mathrm{Na}_{\mathrm{V}} 1.6$ relative to the lipid bilayer after $20 \mathrm{~ns}$ of $\mathrm{MD}$ simulation is shown in Figure 4A. The toxin is again attracted to the periplasmic side of the VS domain, similar to that observed for
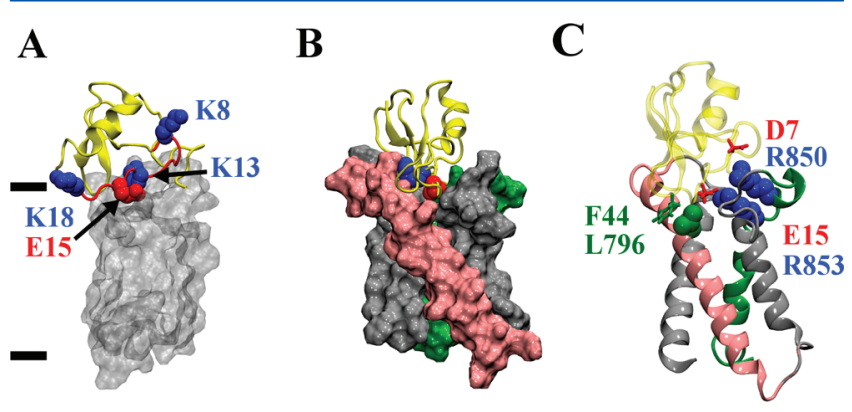

Figure 4. (A) Position of $\mathrm{Cn} 2$ bound to the IIS1-S4 VS domain of $\mathrm{Na}_{\mathrm{V}} 1.6$, relative to the lipid bilayer. (B) $\mathrm{Cn} 2$ bound to the VS domain of $\mathrm{Na}_{\mathrm{V}}$ 1.6. The side chains of two key residues of the toxin, Lys 13 (blue) and Glu15 (red), are highlighted. (C) Cn2 bound to the VS domain of $\mathrm{Na}_{\mathrm{V}} 1.6$ showing three of the key contacts. the Css4-Na 1.2 complex. The two charged residues, Lys13 and Glu15, protrude into the binding groove formed by the S1-S2 and S3-S4 linkers (Figure 4B). The key residue pairs identified from the equilibrated $\mathrm{Cn} 2-\mathrm{Na}_{\mathrm{V}} 1.6$ complex are given in Table 1. We find that the aspartate residue of $\mathrm{Cn} 2$ at the position 7, where it is an asparagine in Css4, is in close proximity to Arg850 (Figure 4C). In addition, the toxin residue Lys13 forms favorable interactions with the glutamate residue of $\mathrm{Na}_{\mathrm{V}} 1.6$ at the position 788, where it is a serine in $\mathrm{Na}_{\mathrm{V}} 1.2$ (see Figure 1B). Thus, two more electrostatic complexes are formed in Cn2$\mathrm{Na}_{\mathrm{V}} 1.6$, compared with the Css4- $\mathrm{Na}_{\mathrm{V}} 1.2$ complex. However, one unfavorable interaction between the residue pair Glu28Glu844 (electrostatic interaction energy of $30 \pm 16 \mathrm{kcal} / \mathrm{mol}$ ) is observed.

PMF Profiles. To verify the bound states of the two toxins predicted from docking and unbiased MD simulations, we construct the PMF profile for the unbinding of the toxins along the bilayer normal and derive the dissociation constant $\left(K_{d}\right)$ for each toxin-VS complex. The converged PMF profiles are displayed in Figure 5. The PMF profiles corresponding to the

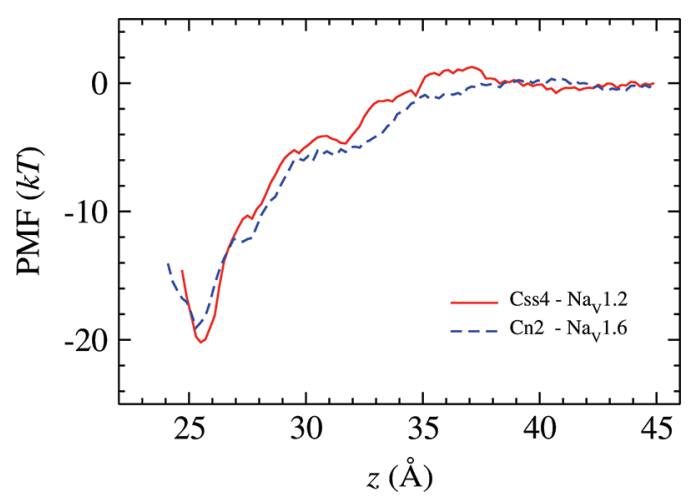

Figure 5. PMF profiles for the unbinding of Css 4 from $\mathrm{Na}_{\mathrm{V}} 1.2$ and $\mathrm{Cn} 2$ from $\mathrm{Na}_{\mathrm{V}}$ 1.6. The maximum errors of both PMF profiles are approximately $0.4 \mathrm{kT}$.

unbinding of the two toxins are very similar, with the depths of the two PMF profiles being $20 \mathrm{kT}$. The $K_{\mathrm{d}}$ values derived from the PMF profiles with eq 1 are $20 \mathrm{nM}$ for Css4- $\mathrm{Na}_{\mathrm{V}} 1.2$ and $70 \mathrm{nM}$ for $\mathrm{Cn} 2-\mathrm{Na}_{\mathrm{V}} 1.6$, respectively. The maximum errors of both PMF profiles measured with the bootstrapping method are $\sim 0.4 \mathrm{kT}$, which lead to an uncertainty of approximately 2.2 fold in the $K_{d}$ values derived.

Experimentally, the $K_{\mathrm{d}}$ value for the binding of Css 4 to $\mathrm{Na}_{V} 1.2$ has been shown to be dependent on the state of the channel. ${ }^{13}$ In the inactivated state of $\mathrm{Na}_{\mathrm{V}} 1.2$, the $K_{\mathrm{d}}$ value is $0.2-3.4 \mathrm{nM}$ for Css 4 , whereas in the resting state the $K_{\mathrm{d}}$ value is substantially higher $(1-5 \mu \mathrm{M}) \cdot{ }^{11,13}$ In the inactivated state, the VS domain may be more exposed to the extracellular space, such that it is easier for toxins to bind. Since the VS domains examined in this work are modeled on the $\mathrm{Na}_{\mathrm{V}} \mathrm{Ab}$ channel crystallized in the inactivated state, ${ }^{4}$ the $K_{d}$ values measured on the inactivated state are used for comparison. Similar $K_{\mathrm{d}}$ values of $0.1-1.0 \mathrm{nM}$ for the binding of Css 4 to the inactivated state have also been reported. ${ }^{15-17}$ Thus, it can be concluded from experiment that the $K_{\mathrm{d}}$ value for the binding of Css4 to inactivated $\mathrm{Na}_{\mathrm{V}} 1.2$ is $\sim 1 \mathrm{nM}$, which is about 1 order of magnitude lower than the value of $20 \mathrm{nM}$ we predicted. The $K_{\mathrm{d}}$ value for $\mathrm{Cn} 2-\mathrm{Na}_{\mathrm{V}} 1.6$ has not been reported. However, the $\mathrm{EC}_{50}$ value for $\mathrm{Cn} 2-\mathrm{Na}_{\mathrm{V}} 1.6$ has been estimated to be about 40 nM. ${ }^{9}$ If assuming that $K_{\mathrm{d}}$ is similar to $\mathrm{EC}_{50}$ in this case, our 
prediction of $70 \mathrm{nM}$ would be in reasonable agreement with experiment. Therefore, the $K_{\mathrm{d}}$ values we predicted are in broad agreement with experiment, suggesting that the models are good representations of the bound states by the toxins and channels.

\section{CONCLUSIONS}

Using computational tools, we reveal the most likely bound complexes of Css4-Nav1.2 and Cn2-Nav1.6 and identify the functional surfaces of the two toxins. We find that the binding groove is formed by the $\mathrm{S} 1-\mathrm{S} 2$ and $\mathrm{S} 3-\mathrm{S} 4$ linkers in both channels, consistent with experiment. ${ }^{11}$ Both toxins wedge into the binding groove of the channel with two central residues, Lys 13 and Glu15, which form favorable electrostatic interactions with the channel. The $K_{d}$ values for the dissociation of the toxins from the channels derived from umbrella sampling simulations are in broad agreement with experiment. Moreover, the functional surface of Css4- $\mathrm{Na}_{V} 1.2$ and $\mathrm{Cn} 2-\mathrm{Na}_{V} 1.6$ we predicted has been found to be critical for the recognition of these toxins by antibodies. ${ }^{39,40}$ Thus, our calculations suggest that the functional surface of antimammalian scorpion $\beta$-toxins is conserved, with Lys13 and Glu15 being the key central residues.

\section{AUTHOR INFORMATION}

\section{Corresponding Author}

*Tel: +61-2-6125-4337. Fax: +61-2-6125-0739. E-mail: rong. chen@anu.edu.au.

Notes

The authors declare no competing financial interest.

\section{ACKNOWLEDGMENTS}

This research was undertaken on the NCI National Facility in Canberra, Australia, which is supported by the Australian Commonwealth Government. This work is supported by grants from the National Health and Medical Research Council of Australia.

\section{REFERENCES}

(1) Catterall, W. A.; Goldin, A. L.; Waxman, S. G. Pharmacol. Rev. 2005, 57, 397-409.

(2) Ren, D.; Navarro, B.; Xu, H.; Yue, L.; Shi, Q.; Clapham, D. E. Science 2001, 294, 2372-2375.

(3) Koishi, R.; Xu, H.; Ren, D.; Navarro, B.; Spiller, B. W.; Shi, Q.; Clapham, D. E. J. Biol. Chem. 2004, 279, 9532-9538.

(4) Payandeh, J.; Scheuer, T.; Zheng, N.; Catterall, W. A. Nature 2011, 475, 353-358.

(5) Catterall, W. A.; Cestele, S.; Yarov-Yarovoy, V.; Yu, F. H.; Konoki, K.; Scheuer, T. Toxicon 2007, 49, 124-141.

(6) Gurevitz, M.; Karbat, I.; Cohen, L.; Ilan, N.; Kahn, R.; Turkov, M.; Stankiewicz, M.; Stühmer, W.; Dong, K.; Gordon, D. Toxicon 2007, 49, 473-489.

(7) Possani, L. D.; Becerril, B.; Delepierre, M.; Tytgat, J. Eur. J. Biochem. 1999, 264, 287-300.

(8) Vazquez, A.; Tapia, J. V.; Eliason, W. K.; Martin, B. M.; Lebreton, F.; Delepierre, M.; Possani, L. D.; Becerril, B. Toxicon 1995, 33, 11611170.

(9) Schiavon, E.; Sacco, T.; Cassulini, R. R.; Gurrola, G.; Tempia, F.; Possani, L. D.; Wanke, E. J. Biol. Chem. 2006, 281, 20326-20337.

(10) Martin, M. F.; Garcia y Perez, L. G.; El Ayeb, M.; Kopeyan, C.; Bechis, G.; Jover, E.; Rochat, H. J. Biol. Chem. 1987, 262, 4452-4459.

(11) Zhang, J. Z.; Yarov-Yarovoy, V.; Scheuer, T.; Karbat, I.; Cohen, L.; Gordon, D.; Gurevitz, M.; Catterall, W. A. J. Biol. Chem. 2011, 286, 33641-33651.
(12) Marcotte, P.; Chen, L. Q.; Kallen, R. G.; Chahine, M. Circ. Res. 1997, 80, 363-369.

(13) Cestèle, S.; Qu, Y.; Rogers, J. C.; Rochat, H.; Scheuer, T.; Catterall, W. A. Neuron 1998, 21, 919-931.

(14) Cestèle, S.; Scheuer, T.; Mantegazza, M.; Rochat, H.; Catterall, W. A. J. Gen. Physiol. 2001, 118, 291-302.

(15) Cohen, L.; Karbat, I.; Gilles, N.; Ilan, N.; Benveniste, M.; Gordon, D.; Gurevitz, M. J. Biol. Chem. 2005, 280, 5045-5053.

(16) Cestèle, S.; Yarov-Yarovoy, V.; Qu, Y.; Sampieri, F.; Scheuer, T.; Catterall, W. A. J. Biol. Chem. 2006, 281, 21332-21344.

(17) Karbat, I.; Ilan, N.; Zhang, J. Z.; Cohen, L.; Kahn, R.; Benveniste, M.; Scheuer, T.; Catterall, W. A.; Gordon, D.; Gurevitz, M. J. Biol. Chem. 2010, 285, 30531-30538.

(18) Cohen, L.; Ilan, N.; Gur, M.; Stuhmer, W.; Gordon, D.; Gurevitz, M. J. Biol. Chem. 2007, 282, 29424-29430.

(19) Leipold, E.; Hansel, A.; Borges, A.; Heinemann, S. H. Mol. Pharmacol. 2006, 70, 340-347.

(20) He, H. Q.; Liu, Z. R.; Dong, B. Q.; Zhang, J. W.; Shu, X. Q.; Zhou, J. J.; Ji, Y. H. PLoS One 2011, 6, e14510.

(21) Song, W.; Du, Y.; Liu, Z.; Luo, N.; Turkov, M.; Gordon, D.; Gurevitz, M.; Goldin, A. L.; Dong, K. J. Biol. Chem. 2011, 286, 1578115788.

(22) Cohen, L.; Karbat, I.; Gilles, N.; Froy, O.; Corzo, G.; Angelovici, R.; Gordon, D.; Gurevitz, M. J. Biol. Chem. 2004, 279, 8206-8211.

(23) Guex, N.; Peitsch, M. C. Electrophoresis 1997, 18, 2714-2723.

(24) Schwede, T.; Kopp, J.; Guex, N.; Peitsch, M. C. Nucleic Acids Res. 2003, 31, 3381-3385.

(25) Arnold, K.; Bordoli, L.; Kopp, J.; Schwede, T. Bioinformatics 2006, 22, 195-201.

(26) Pintar, A.; Possani, L. D.; Delepierre, M. J. Mol. Biol. 1999, 287, $359-367$.

(27) Mintseris, J.; Pierce, B.; Wiehe, K.; Anderson, R.; Chen, R.; Weng, Z. Proteins 2007, 69, 511-520.

(28) Phillips, J. C.; Braun, R.; Wang, W.; Gumbart, J.; Tajkhorshid, E.; Villa, E.; Chipot, C.; Skeel, R. D.; Kalé, L.; Schulten, K. J. Comput. Chem. 2005, 26, 1781-1802.

(29) MacKerell, A. D.; Bashford, D.; Bellott, M.; Dunbrack, R. L.; Evanseck, J. D.; Field, M. J.; Fischer, S.; Gao, J.; Guo, H.; Ha, S.; et al. J. Phys. Chem. B 1998, 102, 3586-3616.

(30) Klauda, J. B.; Venable, R. M.; Freites, J. A.; O'Connor, J. W.; Tobias, D. J.; Mondragon-Ramirez, C.; Vorobyov, I.; MacKerell, A. D., Jr.; Pastor, R. W. J. Phys. Chem. B 2010, 114, 7830-7843.

(31) Jorgensen, W. L.; Chandrasekhar, J.; Madura, J. D.; Impey, R. W.; Klein, M. L. J. Chem. Phys. 1982, 79, 926-935.

(32) Ryckaert, J. P.; Ciccotti, G.; Berendsen, H. J. C. J. Comput. Phys. 1977, 23, 327-341.

(33) Miyamoto, S.; Kollman, P. A. J. Comput. Chem. 1992, 13, 952962.

(34) Kästner, J.; Thiel, W. J. Chem. Phys. 2006, 124, 234106.

(35) Kumar, S.; Bouzida, D.; Swendsen, R. H.; Kollman, P. A.; Rosenberg, J. M. J. Comput. Chem. 1992, 13, 1011-1021.

(36) Chen, R.; Robinson, A.; Gordon, D.; Chung, S. H. Biophys. J. 2011, 101, 2652-2660.

(37) Mantegazza, M.; Cestèle, S. J. Physiol. 2005, 568, 13-30.

(38) Karbat, I.; Turkov, M.; Cohen, L.; Kahn, R.; Gordon, D.; Gurevitz, M.; Frolow, F. J. Mol. Biol. 2007, 366, 586-601.

(39) Calderon-Aranda, E. S.; Selisko, B.; York, E. J.; Gurrola, G. B.; Stewart, J. M.; Possani, L. D. Eur. J. Biochem. 1999, 264, 746-755.

(40) Canul-Tec, J. C.; Riano-Umbarila, L.; Rudino-Pinera, E.; Becerril, B.; Possani, L. D.; Torres-Larios, A. J. Biol. Chem. 2011, 286, 20892-20900. 